\title{
Efectos del cambio climático sobre especies de plantas vasculares del sur de los Andes Centrales: un estudio en el noroeste de Argentina (NOA)
}

\author{
ANA C. Godoy-BüRKI ${ }^{凶}$ \\ Instituto de Botánica Darwinion (IBODA)-CONICET. San Isidro, Buenos Aires, Argentina.
}

\begin{abstract}
RESUMEN. La región de los Andes Tropicales es considerada como una región muy vulnerable al cambio climático porque presenta una alta diversidad y endemismo y porque posee una gran variabilidad altitudinal. Si bien se ha efectuado un gran número de predicciones sobre los cambios que ocurrirán en el clima, pocos estudios evaluaron el impacto que éste tendrá sobre las distribuciones de las especies. En este estudio se estima el efecto del cambio climático sobre la distribución y la conservación de especies endémicas en los años 2050 y 2080 , bajo dos escenarios futuros (A2 y B1), para la región del noroeste argentino, coincidente con la porción sur de los Andes Centrales. Los resultados indican que desde el presente al 2080, el cambio climático en la región del NOA ocasionará un único evento de extinción y modificaciones en los rangos de distribución de las especies endémicas. Además, se observa un desplazamiento generalizado de las especies endémicas hacia zonas de mayor altitud y hacia zonas subtropicales. En consecuencia, se estima que los impactos del cambio climático futuro serán mayores para eco-regiones bajas y menos áridas. Asimismo, el sistema de áreas protegidas del NOA no representará de forma adecuada a la flora endémica en el futuro, bajo ningún escenario, según el objetivo de conservación establecido en el presente trabajo. Estos resultados ponen de manifiesto la necesidad de concentrar los esfuerzos de conservación en las especies y ecosistemas identificados como más vulnerables, con objeto de asegurar la conservación futura de la flora endémica de la parte más sur de los Andes Centrales
\end{abstract}

[Palabras clave: contracción, expansión, rangos de distribución, conservación, endemismo]

\begin{abstract}
AвSTRACT: Effects of climate change on vascular plant species from the southernmost Central Andes: a case study in north-western Argentina (NOA). The Tropical Andes region has been indicated as a highly vulnerable region to climate change for its high diversity and endemism and its great altitudinal variability. While there have been an important number of predictions about the changing climate in the region, few studies have evaluated the impact this will have on the distribution of species. In this paper, we estimated the effect of climate change on the distribution and conservation of endemic species from north-western Argentina (southernmost Central Andes) for the years 2050 and 2080 and under two future scenarios (A2 and B1). According to the results, from the present to 2080, climate change in the region will cause a single extinction event, and changes in all distributional ranges of endemic species. Moreover, a widespread displacement of endemic species towards higher altitudes and subtropical areas is predicted, indicating that the impacts of future climate change will be greater for low and less arid ecoregions. Moreover, the NOA system of protected areas would not be able to represent adequately the endemic flora in the future under any scenario, according to the established target in the present study. These results highlight the need to concentrate conservation efforts on those species and ecosystems identified here as the most vulnerable in order to ensure the future conservation of the endemic flora of the most southern part of the Central Andes.
\end{abstract}

[Keywords: contraction, expansion, distributional range, conservation, endemism]

\section{INTRODUCCIÓN}

La evidencia en los registros fósiles (Davis \& Shaw 2001) y en tendencias observadas recientemente (Hugues 2000; Mc Carty 2001; Walther et al. 2002) demuestra que el cambio climático tiene una influencia profunda en la distribución geográfica de las especies y los ecosistemas (Midgley et al. 2002; Higgins 2007). No obstante, puesto que las especies no poseen la misma habilidad de dispersión y /o de adaptación, el efecto del cambio climático no será uniforme (Daniels \& Veblen 2004).

\section{Editora asociada: Adriana Salvo}

agodoy@darwin.edu.ar
A nivel general y global, se predice que las especies de tierras bajas migrarán hacia áreas más frescas y húmedas cuando estas tierras experimenten aumentos en sus temperaturas (Colwell et al. 2008). Por otro lado, las especies de montaña, en caso de aislamiento, experimentarían extinciones debido a la falta de áreas disponibles para colonizar. Esto ocurriría a menos que dichas especies se adaptaran rápidamente a las condiciones ambientales cambiantes (Colwell et al. 2008; Raxworthy et al. 2008; Larsen et al. 2011).

Recibido: 7 de agosto de 2015

Aceptado: 9 de diciembre de 2015 
Los altos niveles de diversidad y de endemismo, sumado a la variedad de altitudes que presentan los Andes Tropicales, convierten a la región en una zona altamente vulnerable al cambio climático y sus consecuencias (Bush 2002; Malcolm et al. 2006; Vuille et al. 2003). Si bien se han documentado cambios significativos en las temperaturas, regímenes de precipitaciones y en los patrones climáticos estacionales a lo largo de los Andes (Anderson et al. 2011), al presente, pocos estudios han evaluado los efectos y la magnitud que los cambios climáticos tendrán sobre las especies que los habitan (pero véase Herzog et al. 2011).

En los últimos años, la región del noroeste Argentino (NOA), coincidente con una parte de la porción sur de los Andes Centrales, sufrió un aumento de la temperatura media anual de $0.62{ }^{\circ} \mathrm{C}$, además de descensos significativos en la cantidad de precipitaciones $(-4.67 \%$ por década), especialmente en la época invernal (entrelos $22^{\circ} \mathrm{Sy} 28^{\circ} \mathrm{S}$ ) (Gonzales 2009; Marengo et al. 2011). Estos cambios se potencian con la cantidad de actividades antrópicas que se desarrollan en el área (en particular en sus ambientes áridos y semiáridos) y han dado lugar a la pérdida de grandes proporciones de suelo y a modificaciones intensas en su uso (Grau et al. 2005; Izquierdo \& Grau 2009). A pesar de estas observaciones, se desconoce la existencia de estudios que hayan evaluado los impactos del cambio climático sobre las especies en este sector de los Andes Tropicales. La necesidad de generar estimaciones de la vulnerabilidad de las especies al cambio climático resulta crítica para apoyar la planificación de su conservación (Williams et al. 2008). La finalidad del presente trabajo es entonces evaluar cuál será la situación de las especies endémicas del NOA y de las ecoregiones que éstas habitan frente a un cambio climático futuro. Se evaluaron dos periodos de tiempo (2050 y 2080) y se contrastaron dos escenarios climáticos de condiciones opuestas (A2 y B1) (IPCC 2001). Además, se evaluó cual sería el desempeño del actual sistema de áreas protegidas en la conservación de especies ante la ocurrencia un cambio climático.

\section{MAteriales y MÉTODOS}

\section{Área de estudio}

La parte más meridional de los Andes Centrales corresponde al NOA y a la región norte de Chile. El presente estudio comprende el estudio de la porción argentina $\left(520000 \mathrm{~km}^{2}\right.$ ) (Figura 1). Predominan dos tipos de climas: cálido y húmedo (subtropical) en la parte nororiental de la región, y frío y seco (árido) en la parte suroccidental. El gradiente altitudinal va desde los 500 m.s.n.m. al este, hasta valores superiores a 4000 m.s.n.m. al oeste (Cabrera 1976). El gradiente de precipitación resultante de las diferencias altitudinales y la intervención de cadenas montañosas orientadas norte-sur, va desde los 900-3500mm/año en las vertientes orientales de los Andes a menos de $100 \mathrm{~mm} /$ año en la vertiente occidental. La precipitación sigue un régimen monzónico, con lluvias concentradas en verano, de noviembre a febrero (Garreaud et al. 2003). La temperatura oscila dentro de la zona; ocasionalmente, la parte oeste de la porción austral de la región presenta temperaturas bajo cero durante el verano (Cabrera 1976). La región del NOA está representada por siete eco-regiones áridas, semiáridas y sub-húmedas secas (Olson et al. 2001): Estepa Andina Sur , Puna Seca Central Andina, Puna Andina Central, Monte Alto, Yungas Andinas Sur y una pequeña parte del Monte Bajo (Figura 1).

\section{Base de datos}

Puesto que la evidencia sugiere que los patrones históricos del cambio climático influyeron claramente sobre las distribuciones actuales de especies endémicas (Churchill et al. 1995; Killeen et al. 2007), el análisis se basó en el estudio de esas especies. De las 540 especies endémicas identificadas por Aagesen et al. (2012) para el NOA, se trabajó con aquellas que contaban con el número mínimo de ocurrencias necesario para efectuar el modelado de sus distribuciones (268 especies). La distribución futura de las especies se modeló con Maxent (Phillips et al. 2006) que asocia los registros de ocurrencia actuales de las especies con variables climáticas proyectadas para los años 2050 y 2080. El modelo aplicado para la proyección fue el Hadley Centre Coupled Model, versión 3 (HDCM3) (Gordon et al. 2000; Pope et al. 2000) por ser el que mejor representa el clima de Sudamérica (Camilloni \& Bidegain 2005). Los escenarios seleccionados para analizar fueron A2 (alta emisión de gases) y B1 (baja emisión de gases).

\section{Hotspots de endemismo frente al cambio climático}

Para definir los patrones de endemismo de la flora del NOA se creó una grilla del área de 


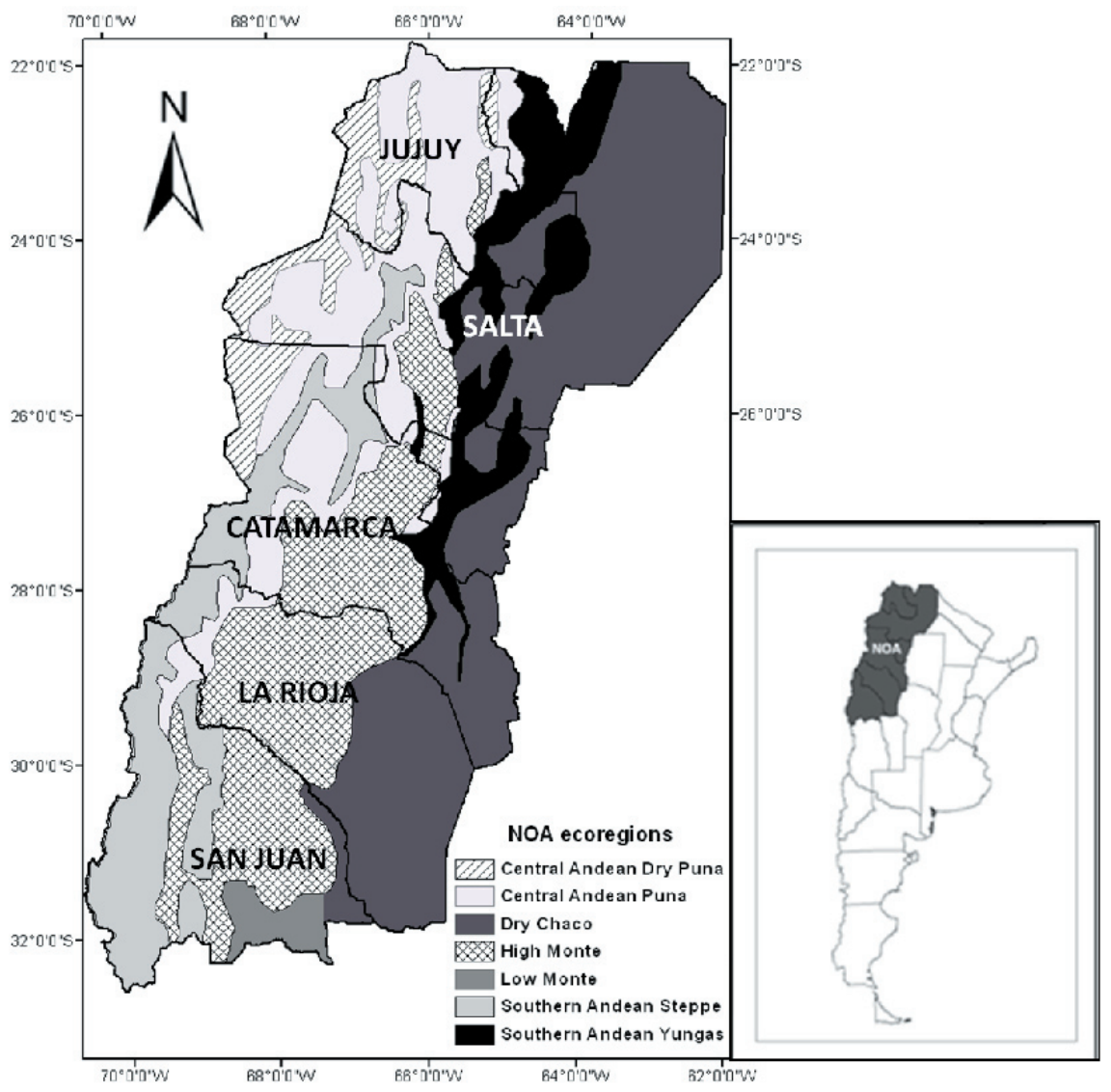

Figura 1. Área de estudio: Noroeste Argentino (NOA) y sus ecorregiones (según Olson et al. [2001]).

Figure 1. Study area: North-western Argentina (NOA) and its ecoregions (sensu Olson et al. [2001]). estudio, con 21058 celdas hexagonales de 25 $\mathrm{km}^{2}$ cada una. Se efectuó el conteo del número de especies endémicas presentes en cada celda para los períodos presente, 2050 (escenarios A2-B1) y 2080 (escenarios A2-B1).

\section{Cambio climático sobre las distribuciones de especies y eco-regiones}

Para el análisis del efecto del cambio climático sobre las distribuciones de las especies, se estimó la superficie de rango geográfico modelada $\left(\mathrm{en}^{\mathrm{km}}{ }^{2}\right.$ ) de cada especie endémica y para los período de tiempo: presente, 2050 (A2-B1) y 2080 (A2-B1), y se efectuaron comparaciones entre las distribuciones potenciales de los diferentes períodos y escenarios. Luego se calculó la proporción en que aumentó (expansión) o se redujo (contracción) cada rango de distribución, en porcentaje.

La vulnerabilidad de las eco-regiones del NOA frente al cambio climático se evaluó sobre la base de los cambios observados en los rangos geográficos de las especies endémicas. En la Tabla 1 se indican las especies y la eco- región a la que representaron. Se consideró como representativa de una eco-región a una especie (abundante/frecuente/dominante) de acuerdo a observaciones realizadas por Cabrera (1976).

\section{Evaluación del estado de conservación futuro de flora endémica}

Para evaluar el estado de conservación de las especies se utilizó el programa MARXAN (Game \& Grantham 2008). Se realizó un análisis GAP para evaluar la eficacia vs. omisiones de la red de reservas actual (Jennings 2000). Los archivos SIG del actual sistema de áreas protegidas (SAP) del NOA(43 áreas protegidas que abarcan una superficie total de de $64348 \mathrm{~km}^{2}$ ) fueron proporcionados por la Administración de Parques Nacionales de Argentina (APN, www.parquesnacionales.go b.ar). Para identificar las especies gap (especies no protegidas por el actual SAP) se estableció como objetivo de conservación ("target") que el actual sistema de reservas debía proteger al menos $25 \%$ del área de distribución de cada una de las especies evaluadas. Este objetivo se planteó siguiendo las conclusiones del trabajo 
Tabla 1. Especies endémicas (de Aagesen et al. 2012) seleccionadas como representativas de cada eco-región.

Table 1. Endemic species (from Aagesen et al. 2012) selected as representative of each ecoregion

\begin{tabular}{ll}
\hline \multicolumn{1}{c}{ Especie } & \multicolumn{1}{c}{ Eco-región } \\
\hline Maytenus cuezzoi & Yungas Andinas Sur \\
Vernonia lipoensis & Yungas Andinas Sur \\
Bulnesia schickendantzii & Monte Alto \\
Plectocarpa rougesii & Monte Alto \\
Nototriche copon & Estepa Andina Sur \\
Astragalus boelckei & Estepa Andina Sur \\
Fabiana punensis & Puna Andina Central \\
Phemeranthus punae & Puna Andina Central \\
\hline
\end{tabular}

de Godoy-Bürki et al. (2014). Se consideró que las especies que alcanzaron el objetivo de conservación propuesto eran especies protegidas, que las especies no representadas dentro de ningún área protegida eran especies gap, y que las que cumplieron con parte del objetivo de conservación eran parcialmente protegidas. El sistema de AP fue considerado efectivo cuando el porcentaje total de especies adecuadamente representadas en base al objetivo fue superior a 60\% (Godoy-Bürki et al. 2014).

\section{Resultados}

Hotspots de endemismo frente al cambio
climático

Los hotspots de endemismo actual se verán afectadas por el cambio climático (Figuras 2 y
3). La descripción de estas áreas fue efectuada por Godoy-Bürki et al. (2014). Bajo el escenario climático A2 se observan cambios en todas las categorías de hotspots, desde las más ricas hasta las más pobres (Figura 2). Se observa un desplazamiento de las especies en sentido este-oeste y sur-norte desde el presente al 2080. Como consecuencia de esta aparente migración de especies, el hotspot de mayor riqueza (>160 especies) parece expandirse. Hacia el 2080 abarcaría los Valles Calchaquíes en Salta (Quebrada de las Conchas, Serranías de Cachi, Quebrada de Escoipe, etc.), las cumbres Calchaquíes en Tucumán y las Sierras del Aconquija y del Cajón, en Catamarca. Los restantes hotspots migrarían hacia el oeste, hacia la cordillera de los Andes (Figura 2). En el 2050, la parte central de Jujuy albergará una gran cantidad de especies, aunque de manera transitoria, ya que para el año 2080 estas especies migrarían hacia las zonas más elevadas de la provincia, en el límite con la cordillera de los Andes (Figura 2). Por otro lado, los hotspots presentes en la provincia de La Rioja y San Juan, ubicados al sur del NOA, presentarían desplazamientos hacia el norte de la región, y se concentrarían en las partes más altas de Catamarca (departamentos de Tinogasta y Antofagasta de la Sierra) y Salta (San Antonio de los Cobres) (Figura 2). Bajo el escenario climático B1 se observa un comportamiento similar en las especies (Figura 3). Los hotspots de mayor endemismo sufrirían un proceso similar al modelado bajo A2. No obstante, hacia el 2080, la provincia de Jujuy presentaría mayor concentración de
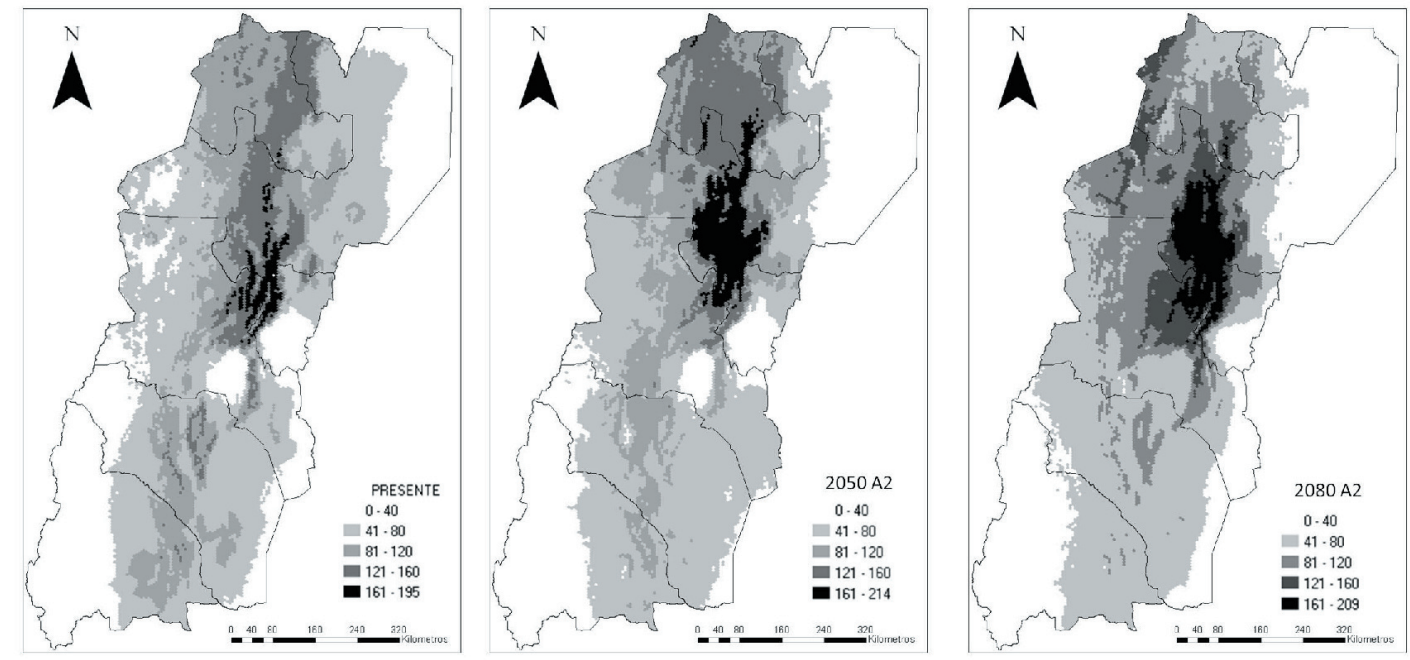

Figura 2. Hotspots de endemismo en el presente, en el 2050 y en el 2080, bajo el escenario climático A2. Figure 2. Endemism hotspots in the present, and in years 2050 and 2080, under the climate scenario A2. 

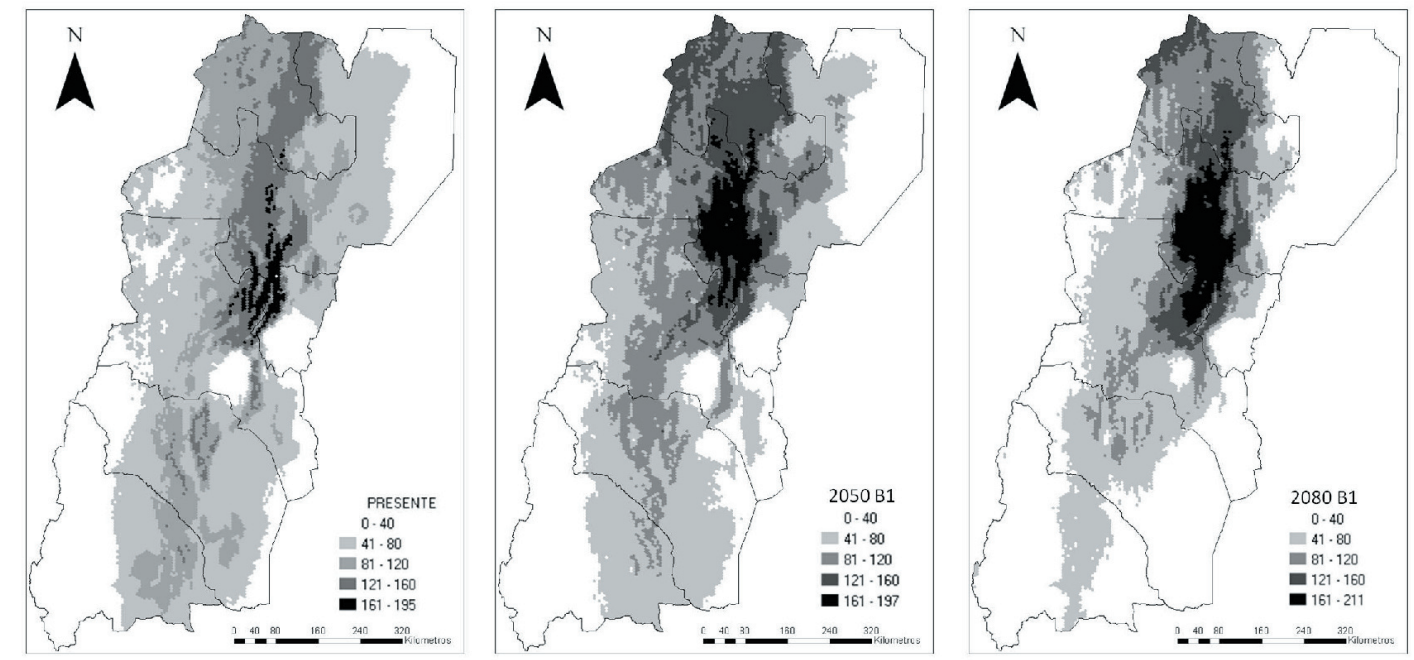

Figura 3. Hotspots de endemismo en el presente, en el 2050 y 2080, bajo el escenario climático B1

Figure 3. Endemism hotspots in the present, 2050 and 2080 period, under the climate scenario B1
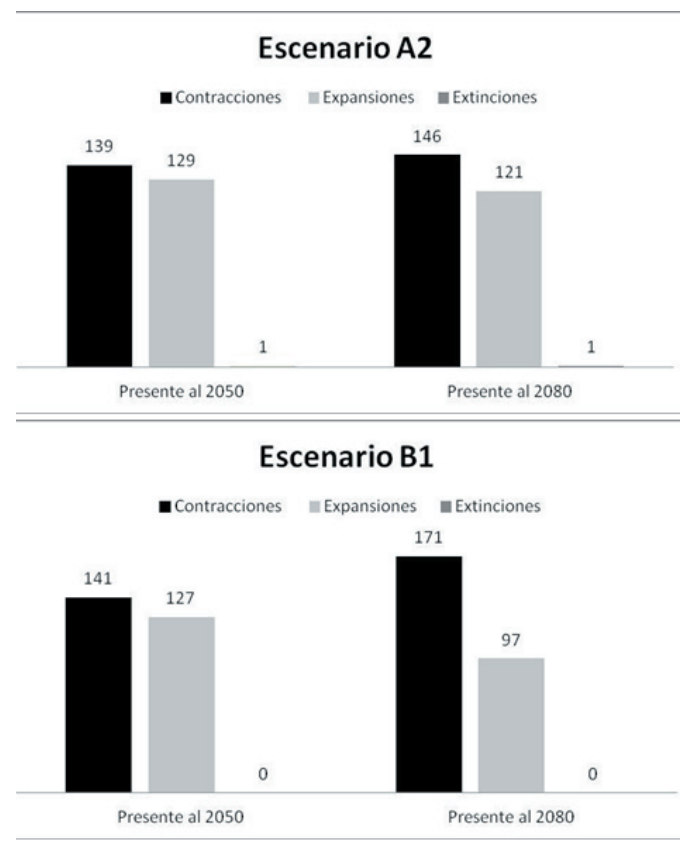

Figura 4. Efectos del cambio climático sobre las especies evaluadas: contrastes entre el número de contracciones, expansiones y/o extinciones en los años 2050 y 2080 (escenarios A2 y B1) vs. la actualidad.

Figure 4. Effects of climate change on species evaluated: contrasts between the number of contractions, expansions and/or extinctions in the years 2050 and 2080 (scenarios A2 and B1) vs. today

especies (120-160 especies) (Figura 3), mientras que la zona de la pre-cordillera de Catamarca presentaría un número mucho menor $(>120$ especies) (Figura 3) con respecto al mismo año pero bajo el escenario opuesto (Figura 2).
Tabla 2. Especies protegidas y parcialmente protegidas en base al objetivo de conservación propuesto en el presente y en los años 2050 y 2080, bajo los escenarios climáticos A2 y B1.

Table 2. Protected species and partially protected species based on the conservation target proposed in the present and in the years 2050 and 2080, under the climate scenarios $\mathrm{A} 2$ and $\mathrm{B} 1$

\begin{tabular}{lccc}
\hline \multicolumn{2}{c}{ Período } & $\begin{array}{c}\text { Especies } \\
\text { protegidas }\end{array}$ & $\begin{array}{c}\text { Especies parcialmente } \\
\text { protegidas }(<25 \%)\end{array}$ \\
\hline \multicolumn{2}{l}{ Presente } & $18(7 \%)$ & $250(93 \%)$ \\
A2 & 2050 & $38(14 \%)$ & $230(86 \%)$ \\
& 2080 & $15(6 \%)$ & $253(94 \%)$ \\
B1 & 2050 & $32(12 \%)$ & $236(88 \%)$ \\
& 2080 & $28(10 \%)$ & $240(90 \%)$ \\
\hline
\end{tabular}

Cambio climático sobre las distribuciones y ecoregiones

El cambio climático simulado generaría contracciones, expansiones y extinciones de especies, representados en la Figura 4. El escenario con mayor número de contracciones sería B1, que a diferencia de A2 tendría 10\% más de especies que sufren una reducción en su distribución. No obstante, bajo el escenario A2 ocurriría el único caso de extinción (Figura 4). La vulnerabilidad de cada eco-región se representa en las Figuras 5 a 8.

\section{Estado de conservación futuro de las especies endémicas}

En la Tabla 2 se exponen los resultados de los niveles de conservación actuales y futuros (2050 y 2080) y bajo diferentes escenarios A2 y 


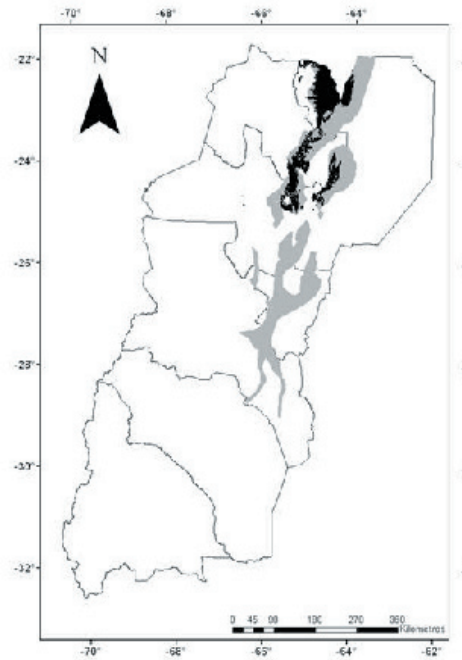

PRESENTE

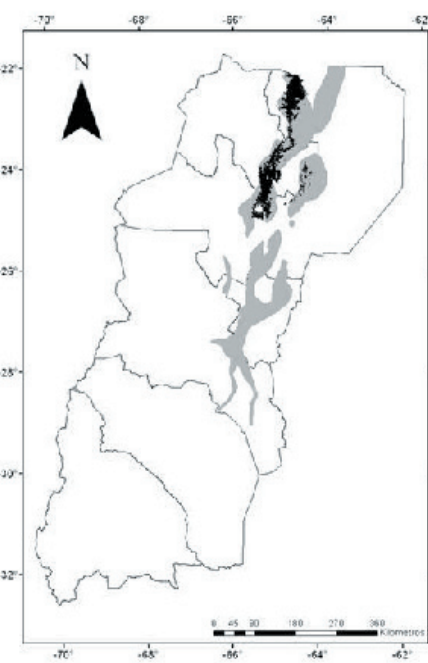

PRESENTE

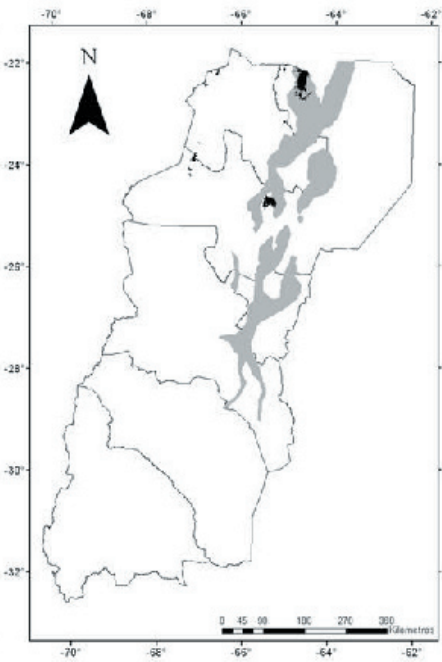

2080 Escenario A2

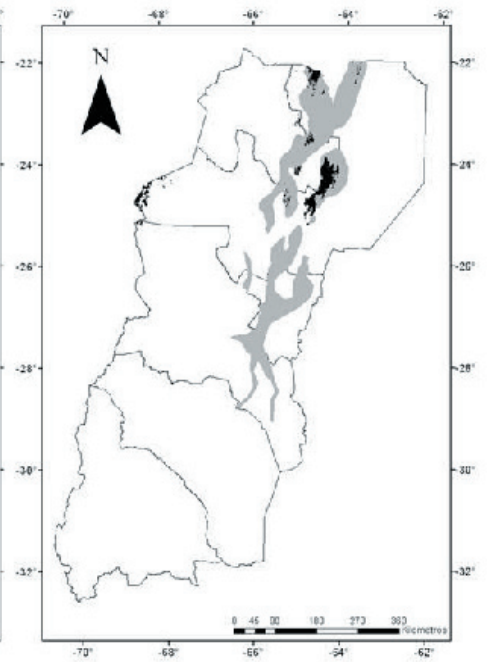

2080 Escenario B1

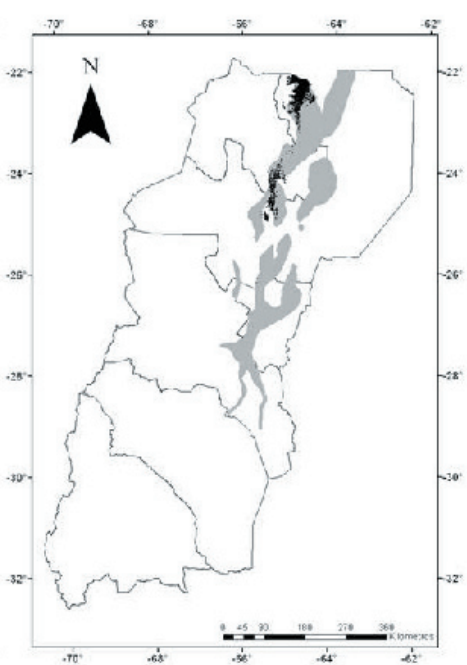

2080 Escenario B1

Figura 5. Distribuciones potenciales (en negro) de Maytenus cuezzoi (arriba) y Vernonia lipoensis (abajo) del presente al 2080, bajo los escenarios climáticos A2 y B1 en la eco-región de las Yungas Andinas Sur (en gris).

Figure 5. Potential distributions (in black) of Maytenus cuezzoi (above) and Vernonia lipoensis (below) from the present to 2080, under A2 and B1 climate scenarios in Southern Andean Yungas ecoregion (in grey)

B1. El sistema de áreas protegidas actual del NOA no sería efectivo en proteger la flora endémica ni en el presente (Godoy-Bürki et al. 2014) (Tabla 2) ni en el futuro, bajo las proyecciones evaluadas (Tabla 2). Los porcentajes de conservación serían similares en ambos escenarios (en base al número de especies protegidas) (ver Tabla 2). El sistema no alcanzaría a cumplir con la representación del objetivo de conservación (25\%) en las proporciones requeridas para considerar al sistema de reservas efectivo ( $>60 \%$ ) en ningún periodo y/o escenario propuesto.

\section{DisCUSIÓN}

Como consecuencia del cambio climático futuro ocurrirían modificaciones en los rangos de distribución de todas las especies evaluadas, y se produciría un único evento de extinción (escenario A2) (Figura 4). Se trataría de la especie Zephyranthes diluta Ravenna (Amaryllidaceae endémica de San Juan) (Zuloaga et al. 2008). Desde el presente hasta el 2080, en ambos escenarios se observa que es mayor el número de especies que contraerían sus rangos de distribución, con respecto al número de especies que lo expandirían (Figura 


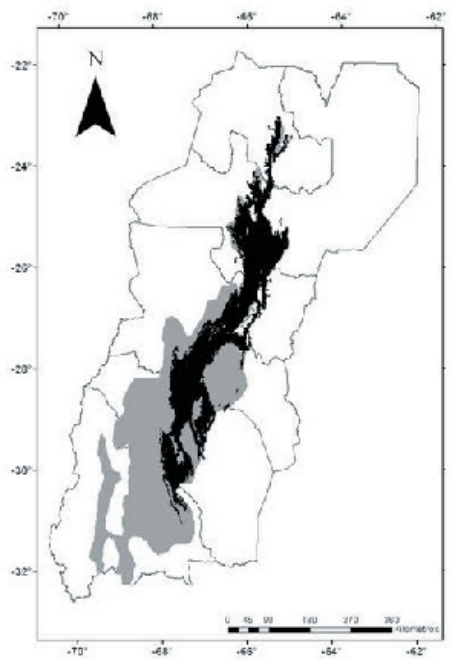

PRESENTE

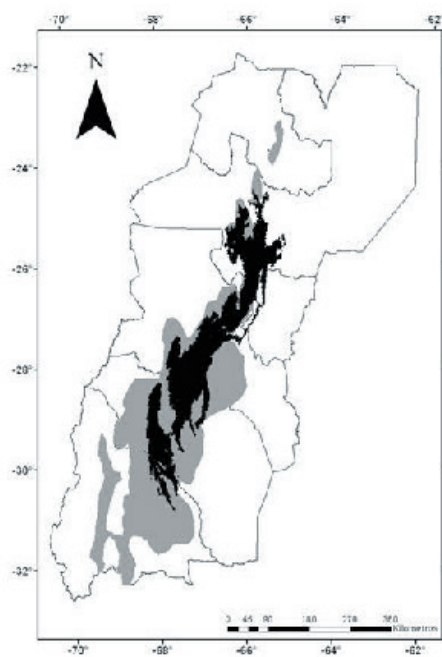

PRESENTE

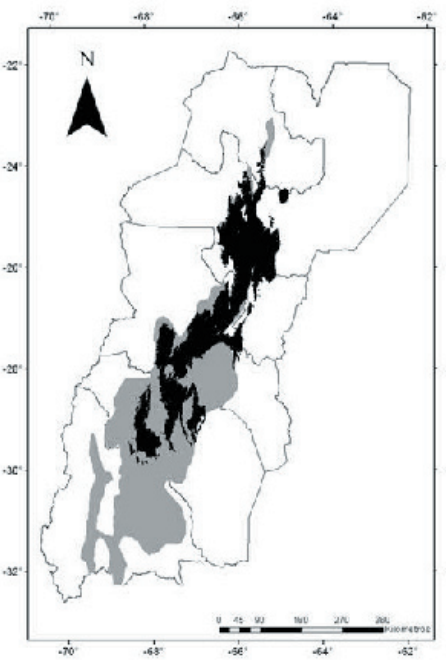

2080 Escenario A2

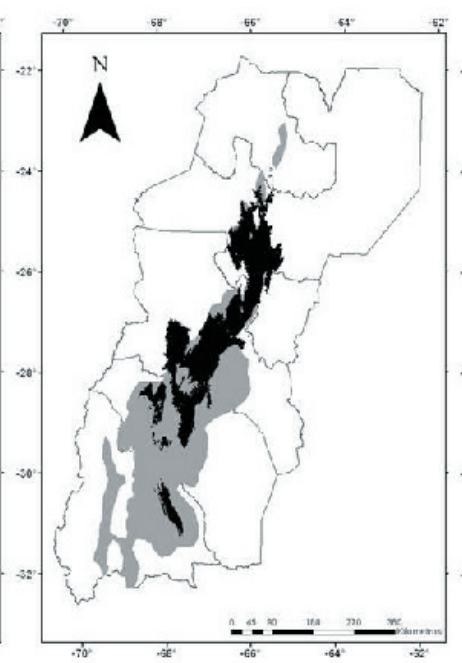

2080 Escenario A2

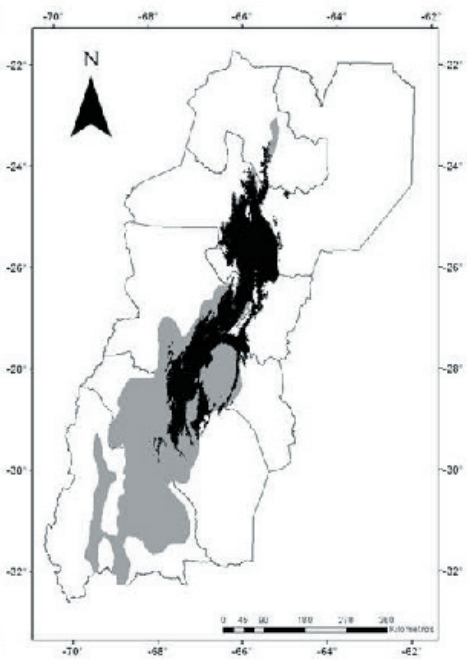

2080 Escenario B1

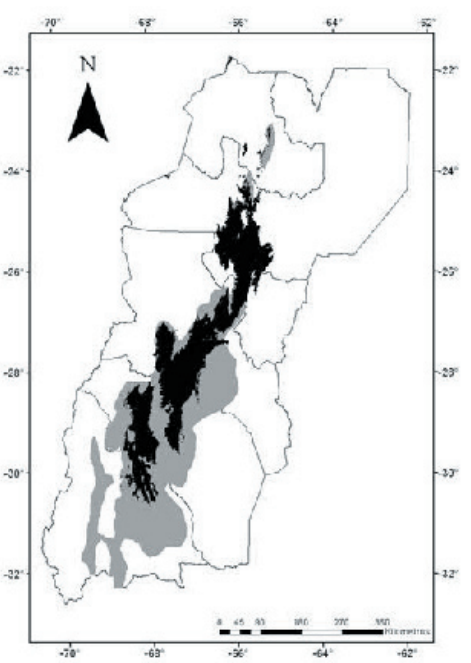

2080 Escenario B1

Figura 6. Distribuciones potenciales Bulnesia schickendantzii (arriba) y Plectocarpa rougesii (abajo), del presente al 2080, bajo el escenario climático A2 y B1, en la eco-región del Monte Alto (en gris).

Figure 6. Potential distributions of Bulnesia schickendantzii (above) and Plectocarpa rougesii (below), from the present to 2080, under A2 and B1 climate scenarios, in High Monte ecoregion (in grey)

4). No obstante, en el escenario climático B1 el cambio sería ligeramente mayor (Figura 4). De las 268 especies endémicas evaluadas, 171 especies $(64 \%)$ reducirían sus rangos geográficos, contra 146 especies (A2) (Figura 4), y más de la mitad de estas reducciones afectaría a más de $50 \%$ de las distribuciones actuales.

Parmesan (2006) presentó evidencia de que muchos taxones montanos han respondido al calentamiento global dispersándose hacia elevaciones mayores. Esta tendencia, con algunas excepciones (Wilson et al. 2007; Moritz et al. 2008), ha sido documentada sobre todo para las plantas leñosas en el límite de su rango altitudinal, o superior (Jump et al. 2009). En nuestro estudio, independientemente de cuáles especies expandirían o reducirían sus distribuciones (Figura 4), se observa una tendencia de las especies endémicas a desplazarse en dirección este-oeste $y$, en menor medida, en dirección sur-norte (desde la actualidad al 2080) (Figuras 4 y 5). La migración de especies ocurriría desde las zonas más bajas (este) hacia las zonas 

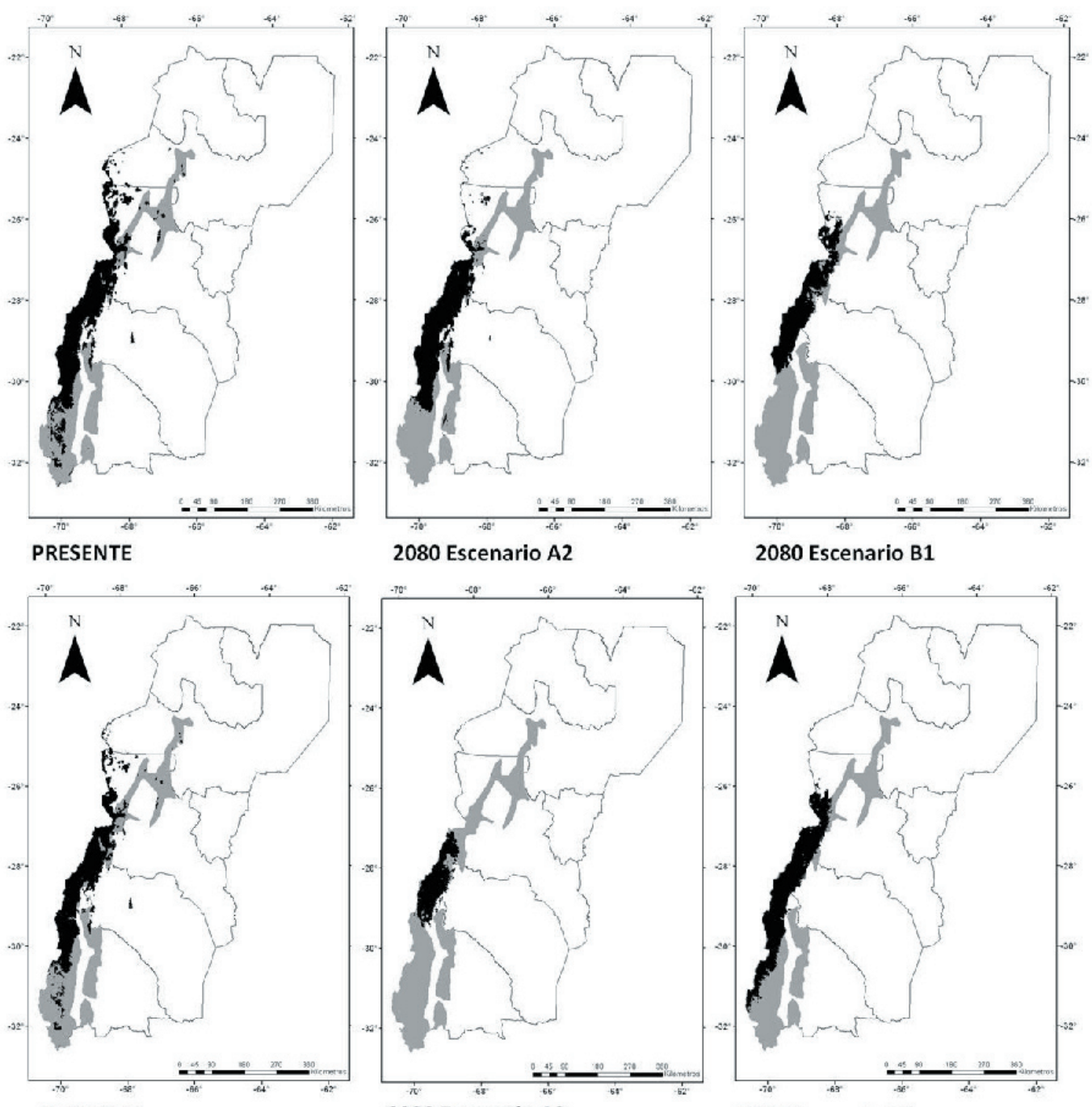

2080 Escenario A2

2080 Escenario B1

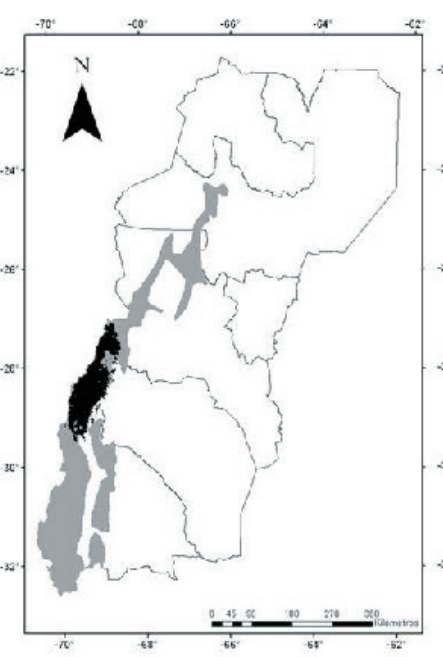

2080 Escenario A2

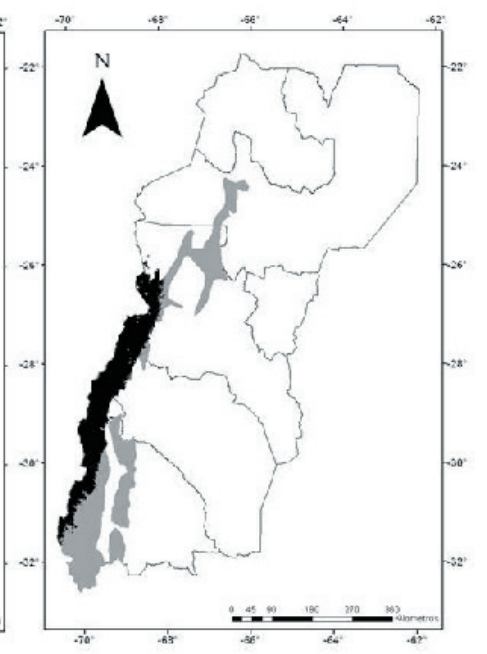

2080 Escenario B1

Figura 7. Distribuciones potenciales de Nototriche copon (arriba) y Astragalus boelckei (abajo) del presente al 2080, bajo el escenario climático A2 y B1, en la eco-región de la Estepa Andina Sur (en gris).

Figure 7. Potential distributions of Nototriche copon (above) and Astragalus boelckei (below) from the present to 2080, under A2 and B1 climate scenarios in Southern Andean Steppe eco-region (in grey).

de mayor altitud (oeste) y hacia zonas más subtropicales (norte). Al parecer, las especies endémicas de las zonas montañosas de mayor elevación lograrían adaptarse a las condiciones ambientales cambiantes sin extinguirse. No obstante, hay que tener en cuenta que los métodos de modelado predictivos empleados no predicen si las especies realmente lograrán alcanzar estos sitios ya que no consideran, por ejemplo, ni los tiempos de adaptación de las especies ni sus habilidades dispersivas. Estas limitaciones e inexactitudes de los modelos condicionan los resultados obtenidos, por lo que deben ser interpretados con precaución (Pearson \& Dawson 2003; Guisan \& Thuiller 2005).
Se estima que el número casi nulo de extinciones y los pocos cambios observados en la flora endémica de la región del NOA como consecuencia del cambio climático podrían asociarse a la heterogeneidad espacial de la región. Estudios sobre la tasa de cambios climáticos a través del tiempo ("climate-change velocity") indican que las áreas heterogéneas amortiguan mejor los cambios climáticos que las zonas llanas (Sandel et al. 2011). Durante épocas de cambios climáticos, las especies de zonas llanas deben moverse más que las especies de regiones heterogéneas para rastrear sus rangos de clima óptimo (Hoorn et al. 2013). En consecuencia, a nivel global, las regiones con una heterogeneidad elevada han 


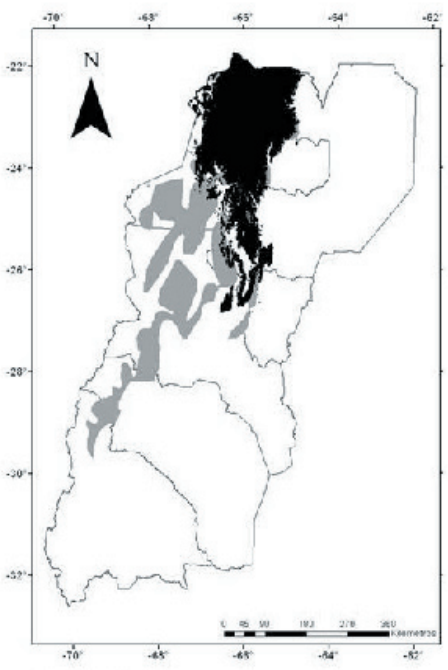

PRESENTE

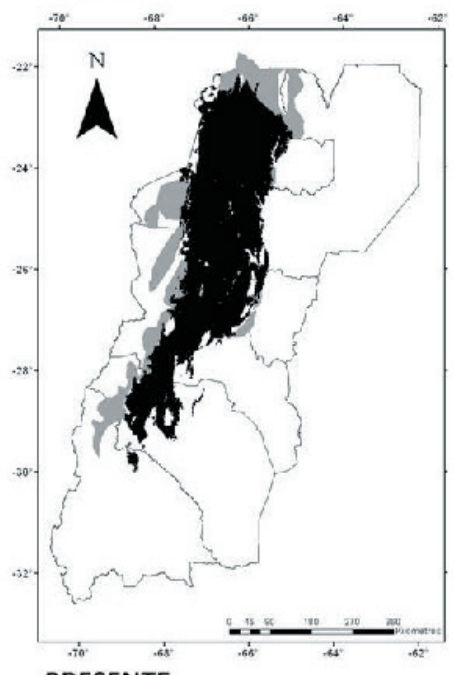

PRESENTE

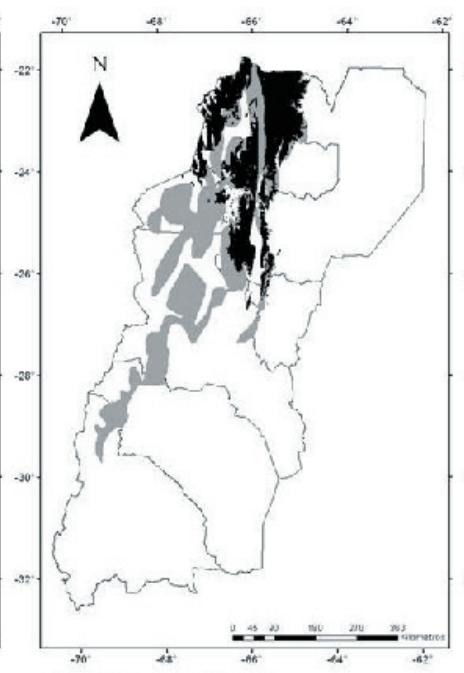

2080 Escenario A2

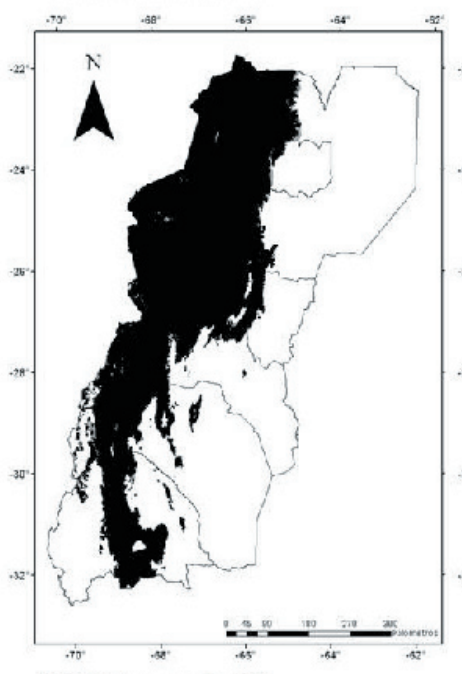

2080 Escenario A2

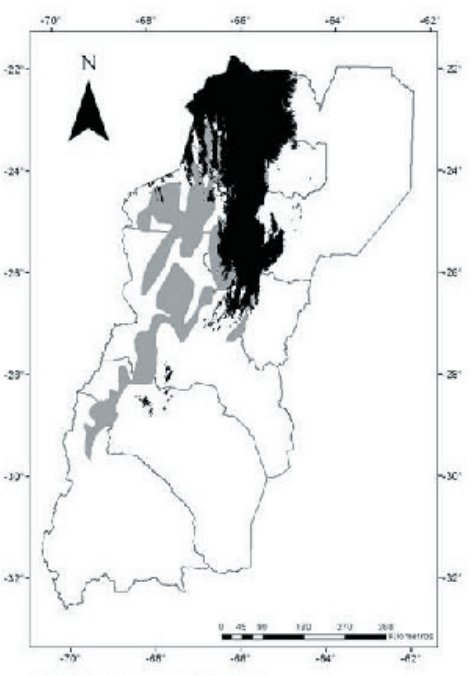

2080 Escenario B1

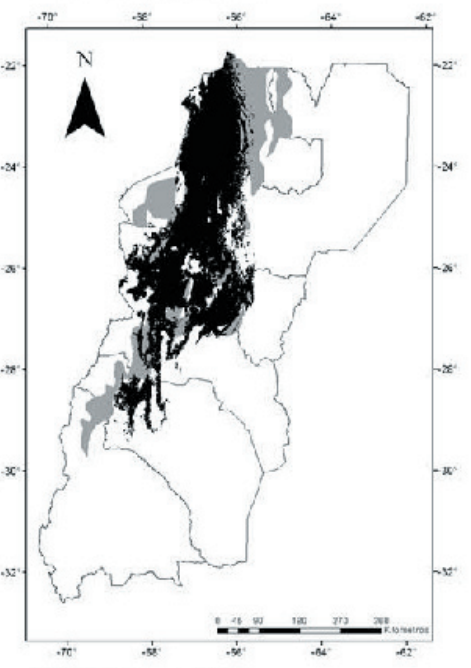

2080 Escenario B1

Figura 8. Distribuciones potenciales (en negro) de Phemeranthus punae (arriba), Fabiana punensis (abajo) del presente al 2080, bajo el escenario climático A2 y B1, en la eco-region de la Puna Andina Central (en gris).

Figure 8. Potential distributions (in black) of Phemeranthus punae (above) and Fabiana punensis (below) from the present to 2080, under A2 and B1 climate scenarios, in the Central Andean Puna ecoregion (in grey).

funcionado como refugios durante los cambios climáticos del cuaternario (Sandel et al. 2011) y probablemente funcionarán como refugios frente a cambios climáticos futuros (Corlett \& Westcott 2013: Diffenbaugh \& Field 2013).

Al evaluar las modificaciones en las distribuciones de las especies endémicas seleccionadas por eco-región (Tabla 1) (figuras 6-9) se observó que el cambio climático afectaría a unos sistemas naturales más que a otros. Las especies estudiadas de las eco-regiones áridas del NOA como el Monte Alto, la Estepa Andina Sur y la Puna Andina Central, no presentarían grandes modificaciones en sus distribuciones, ni diferencias particulares entre escenarios climáticos. Si bien expandirían o reducirían sus rangos de distribución, aparentemente la magnitud de los cambios no superaría el rango de tolerancia climática de estas especies (Figura 6: Monte; Figura 7: Estepa; Figura 8: Puna). La alta resistencia que la flora endémica presenta frente a las condiciones climáticas extremas de estas eco-regiones las convertiría en especies tolerantes a cambios moderados de temperatura (Anderson et al. 2011; Godoy-Bürki, datos no publicados). De hecho, las especies ectotermas de latitudes más altas tienen una tolerancia térmica mayor y viven en climas que actualmente son más frescos que su temperatura fisiológica óptima, por lo que el calentamiento puede, incluso, 
mejorar su condición física (Deutsch et al. 2008). Por lo tanto, se prevé que los impactos del cambio climático futuro sobre las ecoregiones áridas que albergan los centros de endemismo analizados en el presente estudio serían menores.

Las especies de Yungas, por otro lado, sí presentarían cambios notables en sus distribuciones (e.g., Maytenus cuezzoi Legname, Celastraceae [Zuloaga et al. 2008] y Vernonia lipoensis Cabrera, Asteraceae [Zuloaga et al. 2008]), cuyas distribuciones continuas se reducirían a distribuciones disyuntas restringidas (Figura 5). Foster (2001) indicó que los bosques de Yungas son sensibles a los cambios de humedad al punto en que un solo evento de sequía puede causar una mortalidad significativa de plantas. Además, muchas de sus especies se encuentran adaptadas a zonas altitudinales específicas. Se encontró que los elementos áridos de la flora de las Yungas Andinas del Sur y del Chaco Seco están condicionados en mayor medida por la disponibilidad energética, con aumentos en la evapotranspiración potencial de la región; esto genera disminuciones en la diversidad de especies (Godoy-Bürki, datos no publicados). Este efecto negativo también lo produce la precipitación estival, aunque en menor medida (Godoy-Bürki, datos no publicados). En consecuencia, dados los aumentos en las temperaturas y la reducción en las precipitaciones pronosticados por Núñez et al. (2009) para el NOA, y en base a lo observado en el presente estudio, se prevé que los cambios climáticos futuros tendrían un impacto mayor sobre la diversidad de las ecoregiones bajas y menos áridas de la región.

\section{Prioridades de conservación futura de la flora endémica}

En la actualidad, sólo $8 \%$ de los Andes Tropicales se encuentra dentro de áreas protegidas, la cantidad de tierra protegida varía con la altitud y orientación de las laderas, y la desprotección en la parte occidental de los Andes es mayor (Larsen et al. 2011). De acuerdo a los resultados de este trabajo, el sistema de áreas protegidas del NOA no representaría de manera adecuada su flora endémica, ni en el futuro reciente (2050) ni en el futuro lejano (2080) ni en un escenario más (B1) o menos (A2) sostenible con el ambiente. Bajo ningún esquema los niveles de protección alcanzarían el 15\% de efectividad (Tabla 2). En el futuro, $~ 85 \%$ de las especies estaría protegida de manera parcial, y no se sabe si esto garantizaría su permanencia en el largo plazo. De estas especies, 55\% bajo el escenario A2 y menos de $40 \%$ bajo el escenario B1 tendrían bajo protección menos del 15\% de sus rangos de distribución.

Godoy-Bürki et al. (2014) observaron que la falta de congruencia entre los hotspots de endemismo y de riqueza a nivel regional, junto con la tendencia de seleccionar áreas "ad hoc" o basándose en riqueza, generan un sistema de reservas inefectivo en proteger la flora endémica de la región del NOA. En un futuro climático cambiante, la situación no presentaría grandes diferencias (Tabla 2). La falta de congruencia espacial permanecería y podría acentuarse como consecuencia de los desplazamientos de las especies endémicas hacia zonas con condiciones adecuadas para su supervivencia. De acuerdo a los resultados, la región más apta sería la parte más central del NOA en las vertientes occidentales de la Sierra del Aconquija junto a las Cumbres y Valles Calchaquíes (Figuras 2 y 3). Considerando el tiempo que demandaría agregar nuevas áreas protegidas o expandir las existentes, es recomendable la incorporación de las nuevas unidades aplicando al endemismo como criterio de selección (Godoy-Bürki et al. 2014) y teniendo en cuenta los posibles cambios futuros en las distribuciones de las especies en respuesta al cambio climático. A modo de prevención, mayores esfuerzos de conservación deberían dirigirse a las especies de eco-regiones más vulnerables y/o a la creación de una mayor cantidad de reservas dentro de las zonas donde las especies tenderían a desplazarse. Asimismo, deberían incrementarse la cantidad de esfuerzos, tiempo y recursos destinados a disminuir la velocidad de los cambios climáticos predichos para evitar que avancen con mayor velocidad que la flora endémica de la región.

Agradecimientos.Quisiera agradecer a mis directores Lone Aagesen y Fernando Zuloaga por el apoyo y aliento constante. Al CONICET por la beca doctoral otorgada que me permitió efectuar este trabajo y al IBODA, mi lugar de trabajo. 


\section{REFERENCIAS}

Aagesen, L; MJ Bena; S Nomdedeu; A PanizzA; R López; Et Al. 2012. Areas of endemism in the Southern Central Andes. Darwiniana, 50:218-251.

Anderson, EP; J Marengo; R Villalba; S Hallox; BE Young; Et Al. 2011. Consequences of climate change for ecosystems and ecosystem services in the tropical Andes (Parte 1.1). Pp. 1-5 en: Herzog, SK; R Martínez; PM Jorgensen \& H Tiessen (eds.). Climate Change and Biodiversity in the Tropical Andes. Climate change and biodiversity in the tropical Andes. Inter-American Institute for Global Change Research and Scientific Committee on Problems of the Environment (SCOPE), Sao José dos Campos. Pp. 348.

Bush, MB. 2002. Distributional change and conservation on the Andean flank: a palaeoecological perspective. Glob. Ecol. Biogeogr., 11:463-473.

Cabrera, AL. 1976. Regiones fitogeográficas argentinas. Enciclopedia Argentina de Agricultura y Jardinería. Tomo 2. Fascículo 1. ACME, Buenos Aires. Pp. 1-85.

Camilloni, I \& M Bidegain. 2005. Escenarios Climáticos para el siglo XXI. Capítulo 4. Pp. 33-39 en: Barros, V; A Menéndez \& G Nagy (eds.). El Cambio Climático en el Río de la Plata. Editorial CIMA, Buenos Aires.

Colwell, RK; G Brehm; CL Cardelús; AC Gilman \& JT Longino. 2008. Global warming, elevational range shifts, and lowland biotic attrition in the wet tropics. Science, 322:258-261.

Corlett, RT \& DA Westcott. 2013. Will plant movements keep up with climate change? Trends Ecol. Evol., 28:482488.

DANiELS, LD \& TT Veblen. 2004. Spatiotemporal influences of climate on altitudinal treeline in northern Patagonia. Ecol., 85:1284-1296.

DAVIS, MB \& RG SHAW. 2001. Range shifts and adaptive responses to Quaternary climate change. Science., 292:673679.

Deutsch, CA; JJ Tewksbury; RB Huey; KS Sheldon; CK Ghalambor; et al. 2008. Impacts of climate warming on terrestrial ectotherms across latitude. PNAS., 105:6668-6672.

Diffenbaugh, NS \& CB Field. 2013. Changes in ecologically critical terrestrial climate conditions. Science., 341:486492.

FOSTER, P. 2001. The potential negative impacts of global change on tropical montane cloud forests. Earth-Sci. Rev., 55: 73-106.

Game, ET \& HS Grantham. 2008. Marxan User Manual: For Marxan version 1.8.10. University of Queensland, Australia, and Pacific Marine Analysis and Research Association, Vancouver, British Columbia, Canada.

Garreaud, R; M Vuille \& Clement AC. 2003. The climate of the Altiplano: observed current conditions and mechanism of past changes. Paleogeogr. Palaeoclimatol. Palaeoecol., 194:1-18.

Godoy-Bürki, AC; P Ortega-Baes; J Sajama; L Aagesen. 2014. Conservation priorities in the Southern Central Andes: mismatch between endemism and diversity hotspots in the regional flora. Biodiv. Conserv., 23:81-107.

Gonzáles, JA. 2009. Climatic change and other anthropogenic activities are affecting environmental services on the Argentina Northwest (ANW). Earth Environ. Sci., 6:1-2.

Gordon, C; C Cooper; CA Senior; H Banks; JM Gregory; et Al. 2000. The simulation of SST, sea ice extents and ocean heat transports in a version of the Hadley Centre coupled model without flux adjustments. Clim. Dyn., 16:147-168.

Grau, RH; IN Gasparri \& MT Aide. 2005. Agriculture expansion and deforestation in seasonally dry forests of northwest Argentina. Environ. Conserv., 32:140-148.

Guisan, A \& W Thuiller. 2005. Predicting species distribution: offering more than simple habitat models. Ecol. Lett., 8: 993-1009.

Herzog, SK; R Martínez; PM Jorgensen \& H Tiessen. 2011. Climate change and biodiversity in the tropical Andes. São José dos Campos: Inter-American Institute for Global Change Research.

Higgins, PAT. 2007. Biodiversity loss under existing land use and climate change: an illustration using northern South America. Glob. Ecol. Biogeog., 16:197-204.

Hoorn, C; V Mosbrugger; A Mulch \& A Antonelli. 2013. Biodiversity from mountain building. Nat. Geosci., 6:154154.

IPCC (Intergubernamental Panel on Climate Change). 2001. Climate Change 2001, Impacts, Adaptation, and Vulnerability. Contribution of Working Group II to the IPCC Third Assessment Report (TAR). Cambridge University Press, UK.

IzQUiERDo, AE \& HR Grau. 2009. Agriculture adjustment, land-use transition and protected areas in Northwestern Argentina. J. Environ. Manag., 90:858-865

Jump, AS; C MÁTYÁs \& J PeÑuelas . 2009 The altitude-for-latitude disparity in range retractions of woody species. Trends Ecol. Evol., 24:694-701.

Killeen, TJ; M Douglas; T Consiglio; PM Jørgensen \& J Mejía. 2007. Dry spots and wet spots in the Andean hotspot. J Biogeogr 34:1357-1373.

Larsen, TH; G Brehm; H Navarrete; P Franco; H Gómez; et al. 2011. Range shifts and extinctions driven by climate change in the tropical Andes: synthesis and directions. Chapter 3. Pp. 47-67 en: Herzog, SK; R Martínez; PM Jorgensen \& H Tiessen (eds.). Climate Change and biodiversity in the tropical Andes. Inter-American Institute of Global Change Research and Scientific Committee on Problems of the Environment (SCOPE).

Malcolm, JR; C Liu; RP Neilson; L Hansen \& LE Hannah. 2006. Global warming and extinctions of endemic species from biodiversity hotspots. Conserv. Biol., 20:538-548. 
Marengo, JA; JD Pabón; A Díaz; G Rosas; G Ávalos; et AL. 2011. Climate change: evidence and future scenarios for the Andean region. Pp. 110-127 en: Herzog, SK; R Martínez; PM Jorgensen \& H Tiessen (eds.). Climate Change and biodiversity in the tropical Andes. Inter-American Institute of Global Change Research and Scientific Committee on Problems of the Environment (SCOPE).

Midgley, GF; L Hannah; D Millar; MC Rutherford \& LW Powrie. 2002. Assessing the vulnerability of species richness to anthropogenic climate change in a biodiversity hotspot. Glob. Ecol. Biogeogr., 11:445-451.

Moritz, C; JL Patton; CJ Conroy; JL Parra; GC White; et AL. 2008. Impact of a century of climate change on smallmammal communities in Yosemite National Park, USA. Science, 322:261-264.

NúÑEZ, MN; SA Solman \& MF CABRÉ. 2009. Regional climate change experiments over southern South America. II: Climate change scenarios in the late twenty-first century. Clim. Dyn., 32:1081-1095.

Olson, DM; E Dinerstein; ED Wikramanayake; ND Burgess; GVN Powell; Et AL. 2001. Terrestrial ecoregions of the World: a new map of life on Earth. BioSci., 51:933-938.

PARMESAn, C. 2006. Ecological and evolutionary responses to recent climate change. Annu. Rev. Ecol. Evol. Syst., 37: 637-669.

PeARSON, RG \& TP DAWSON. 2003. Predicting the impacts of climate change on the distribution of species: are bioclimate envelope models useful? Glob. Ecol. Biogeogr., 12:361-371.

Phillips, S; R ANDERson \& R SCHAPIRE. 2006. Maximum entropy modelling of species geographic distributions. Ecol. Model., 190:231-259.

Pope, VD; ML Gallani; PR Rowntree \& RA StRATTON. 2000. The impact of new physical parametrizations in the Hadley Centre climate model HadAM3. Clim. Dyn., 16:123-146.

RAXWORTHY, CJ; RG PEARSON; N RABIBISOA; AM RAKOTONDRAZAFY; JB RAMANAMANJATO; ET AL. 2008. Extinction vulnerability of tropical montane endemism from warming and upslope displacement: a preliminary appraisal for the highest massif in Madagascar. Glob. Chang. Biol., 14:1703-1720.

SAndel, B; L Arge; B DalsGaArD; RG Davies; KJ Gaston; ET AL. 2011. The influence of Late Quaternary climate-change velocity on species endemism. Science, 334:660-664.

Vuille, M; RS Bradley; M Werner \& F Keimig. 2003. 20th century climate change in the tropical Andes: observations and model results. Pp. 75-99 en: Climate Variability and Change in High Elevation Regions: Past, Present Future. Springer, Netherlands.

Walther, GR; E Post; P Convey; A Menzel; C Parmesan; et AL. 2002. Ecological responses to recent climate change. Nature, 416:389-395.

Wilson, RJ; D Gutiérrez; J Gutiérrez \& VJ Monserrat. 2007. An elevational shift in butterfly species richness and composition accompanying recent climate change. Glob. Chang. Biol., 13:1873-1887.

Williams, SE; LP Shoo; JL IsaAC; AA Hoffmann \& G Langham. 2008. Towards an integrated framework for assessing the vulnerability of species to climate change. PLOS Biol., 6:325.

Zuloaga, FO; O Morrone \& MJ Belgrano. 2008. Catálogo de las Plantas Vasculares del Cono Sur. Monogr. Syst. Bot. Missouri Bot. Gard., 107:609-967 